\title{
THE PREVALENCE AND FEATURES OF ULTRASOUND PULMONARY ARTERIAL HYPERTENSION IN PATIENTS WITH SYSTEMIC LUPUS ERYTHEMATOSUS
}

\author{
Guşetu Gabriel, Petcu Ana, Rinzis Mirela, Matuz Roxana, Mureșan Lucian, Cismaru Gabriel, \\ Roşu Radu, Irsay Laszlo, Dogaru Gabriela, Pop Dana, Zdrenghea Dumitru, Rednic Simona \\ University of Medicine and Pharmacy "Iuliu Hatieganu" Cluj-Napoca
}

\begin{abstract}
.
Background. Connective tissue diseases (CTDs) are an important cause of pulmonary arterial hypertension $(\mathrm{PAH})$, which leads to worsening of prognosis especially in patients with systemic lupus erythematosus (SLE) and systemic sclerosis. However, studies on the prevalence of PAH in SLE scarce; our aim is to assess the prevalence and characteristics of PAH in a series of SLE inpatients of a tertiary Romanian SLE Center.

Methods. The study included 54 consecutive SLE patients with a regular follow-up at the Department of Rheumatology Cluj-Napoca. The patients underwent physical examination and transthoracic echocardiography to evaluate systolic pulmonary arterial pressure (sPAP), left ventricular performance, and the presence of valvular heart disease. Patient characteristic, cumulative organ damage and laboratory were retrieved by medical chart review.

Results. Within the cohort (mean age $43.7 \pm 12.4$ years, $90.8 \%$ women, median duration of disease 7 years), $11(20.3 \%)$ patients were diagnosed with PAH, the majority of which (63.6\%) were categorized as mild. The mean sPAP value was $45.54 \mathrm{mmHg}$ and was associated with a history thromboembolic events $(p=0.0067)$, antiphospholipid antibodies (aPL) $(p=0.039)$, and cumulative organ damage $(\mathrm{p}=0.001)$. No significant associations with disease duration, Raynaud's phenomenon, pericardial effusion or SLE-associated autoantibodies were found. Left ventricular diastolic dysfunction (LVDD) occurred more frequent in patients with PAH $(\mathrm{p}=0.008)$.

Conclusion. Patients with SLE have an increased prevalence of PAH, which is generally asymptomatic and of low severity. PAH is associated with cumulative organ damage, LVDD, and antiphospholipid syndrome (likely reflecting secondary PAH to pulmonary embolism), but not with the disease duration and the Raynaud's phenomenon or SLE specific autoantibodies.
\end{abstract}

Keywords: systemic lupus erythematosus, pulmonary arterial hypertension, antiphospholipid syndrome, echocardiography

\section{Introduction}

Connective tissue diseases (CTDs) are an important cause of pulmonary arterial hypertension (PAH) (1) and its evolution is less favorable than idiopathic PAH (IPAH) in some cases, with higher mortality rates and a poor response to specific vasodilator therapy (2). As shown in a substudy of the REVEAL registry (3), PAH associated with systemic lupus erythematosus (SLE) has a better prognosis than in systemic sclerosis ( $\mathrm{SSc}$ ), therefore the profile and the prevalence of PAH in SLE patients have been less studied; apart from this, the wide range of reported $\mathrm{PAH}$ prevalence (between 1 and 40\%) in lupus derives from the non-specific and variable clinical manifestations (dyspnea, fatigue, chest pain, palpitations, even syncope), due to which the diagnosis of PAH can be delayed or missed. However its onset, regardless of the mechanism, definitely alters the functional status of patients and negatively impacts the general outcome in SLE patients.

The early diagnosis and follow-up of PAH might more accurately allow the time at which conventional or specific vasodilator therapy (which has proved its efficiency in IPAH and SSc-asociated PAH) should be initiated in patients with SLE. 
One of the methods for PAH diagnosis is echocardiography, enabling the evaluation of systolic pulmonary arterial pressure (sPAP) (4, 5 ), which has long been used in other cardiac diseases known to result in PAH (valvulopathy, congenital heart disease, cardiomyopathy) and which seems to be feasible in CTDs, although the gold standard remains right heart catheterization (difficult to use as routine because of the costs and procedure's risks).

Given that this aspect has been less studied in Romania, this research aims to evaluate the prevalence and the features of PAH diagnosed by ultrasound in a series of SLE patients from the Clinic of Rheumatology Cluj-Napoca.

\section{Material and method}

Patients. The study included 54 consecutive patients in follow at the Department of Rheumatology Cluj-Napoca and 20 age and sex matched healthy controls. All SLE patients fulfilled at least 4 of the American College of Rheumatology classification criteria (6). Patients with any conditions that could affect pulmonary artery pressure (as COPD, asthma, pulmonary fibrosis due to occupational dusts or diagnosed prior to SLE, cardiomyopathies, hemodynamically significant valvular heart diseases, treatment with drugs known to induce pulmonary hypertension (1) and liver cirrhosis) were excluded.

Study design. The patients were evaluated clinically and in terms of personal and family history of cardiovascular disease; laboratory data and findings related to the functional SLICC score (the Systemic Lupus International Collaborating Clinics damage index), antiphospholipid syndrome (APS) components and SLE specific autoantibodies were retrieved by medical chart review. All patients and the subjects of the control group were undergoing a transthoracic echocardiography in the Cardiology Department of the Clinical Rehabilitation Hospital; on the left lateral decubitus, standard 2D measurements, calculation of the left ventricular ejection fraction (LVEF) using the Simpson method, color Doppler (valvular regurgitation assessment) as well as continuous wave (pressure gradient measurement by modified Bernoulli equation) and pulsed wave Doppler echocardiography (diastolic mitral flow assessment) were performed, using an Esaote MyLab50 XVision device with a 3.5 $\mathrm{MHz}$ transducer.

Systolic pulmonary arterial pressure was assessed by measuring the peak systolic pressure gradient of the tricuspid regurgitation flow using the simplified Bernoulli equation (4). Right atrial pressure was estimated at 5 $\mathrm{mmHg}$ if a normal diameter $(<20 \mathrm{~mm})$ and an inspiratory collapse $>50 \%$ of the inferior vena cava was registered. Systolic pressure of the right ventricle (RV) was obtained by adding the systolic tricuspid pressure gradient and right atrium pressure and was considered equal to SPAP, in the absence of right ventricular outflow tract or pulmonary valve obstruction. PAH was diagnosed if sPAP $>30 \mathrm{mmHg}$. If tricuspid regurgitation could not be evidenced but there were no signs of RV dilation or RV systolic dysfunction, sPAP was considered to be normal (7). RV was considered dilated if the $\mathrm{RV}$ area was larger than the left ventricle (LV) area; a tricuspid annular systolic excursion (TAPSE) $<14 \mathrm{~mm}$ was considered RV systolic dysfunction. PAH was classified as mild up to $45 \mathrm{mmHg}$, moderate up to $60 \mathrm{mmHg}$, and severe at $60 \mathrm{mmHg}$ or more (5).

The study was designed in accordance with the Code of Ethics of the World Health Organization (Declaration of Helsinki) for experiments involving humans. All the patients were informed about the study procedures and signed their consent to participate in the study.

Statistical analysis was carried out using the OpenEpi (www.OpenEpi.com) and SPSS for Windows (v 14.0, IBM Corporation, USA) software programs. The results were presented as numbers and percentages for qualitative variables and, for quantitative variables as mean \pm standard deviation; for nonparametric variables, median and range was used. In order to assess the normal (parametric) distribution of continuous numerical variables, the Kolmogorov-Smirnov test was used. To compare the qualitative variables (frequencies), the chi-squared $\left(\chi^{2}\right)$ test was used. The analysis of quantitative 
variables with normal distribution was carried out using the Student t-test (t); for nonparametric variables the Mann-Whitney test was applied. A value of $p<0.05$ was considered statistically significant.

\section{Results}

Demographic and personal history data are presented in table I.

The mean age of the patients was 43.7 \pm 12.4 years, and 49 of these $(90.8 \%)$ were women. The duration of the disease ranged between 0.3 and 43 years, with a median value of 7 years. Age at diagnosis ranged between 17 and 58 years. Both classical cardiovascular risk factors and clinically manifest atherosclerotic disease were present in a higher proportion in the study group, but statistical significance was not reached.

In the studied group, 12 patients $(22.2 \%)$ had no tricuspid regurgitation, but sPAP was considered normal in the context of a RV with normal size and kinetics; pulmonary hypertension was present in 11 patients $(20.3 \%)$, a higher percentage than in the control group $(5.5 \%)$; in the majority of the patients ( 7 patients - 63.6\%), this was categorized as mild (fig. 1). In addition, the mean sPAP value in the group of patients with SLE, although within normal limits, was significantly higher than the mean value registered in the control group $(p=0.0003$, table I).

As it was shown above, higher values of sPAP were not asociated by the suggestive symptoms, which were found in a comparable number of patients in the subgroup with and without PAH. The Raynaud's phenomenon wasn't much more frequent in the subgroup of patients with pulmonary hypertension (table II).
However, in the PAH group there were more thrombotic events ( $72 \%$ vs $2.3 \%, \mathrm{p}<0.01$ - table II), even without clinical clues of pulmonary (micro)embolism - dyspnea, cough, chest pain/discomfort, tachycardia, fever/subfebrility. For the rest of clinical data, such as the presence of cardiovascular risk factors, clinically manifest atherosclerotic disease, osteoarticular or muscular involvement, and even renal or neurological impairment, their proportion was not significantly different between the two subgroups. Only the value of the SLICC, as a global damage index was significantly higher in the subgroup of patients with PAH $(p=$ 0.001 ).

Of laboratory data (table II), antiphospholipid antibodies (aPL) were in a significantly higher proportion in the subgroup of patients with PAH $(p=0.039)$. In contrast, patients in the subgroup with pulmonary hypertension did not have higher rate of anti-dsDNA or renal function impairment. In accordance with the study design, LVEF was normal in both the entire group $(60 \pm 10.34 \%)$ and the two subgroups (table III), but LV diastolic dysfunction was found in a higher number of patients with PAH (90.9\% vs $25.5 \%)$, being classified in the majority of the cases $(80 \%)$ as having $\mathrm{I}^{\text {st }}$ degree of severity(8). Ten patients $(18.5 \%)$ had hemodynamically insignificant valvular heart disease: mild mitral regurgitation - 9 patients $(16.7 \%), 1$ patient with moderate mitral regurgitation $(1.8 \%)$, mild aortic regurgitation - 2 patients $(3.7 \%)$. No significant differences in valvular regurgitation or pericardial effusion were found between the subgroups with and without PAH. 
Table I. Demographic, clinical features and average sPAP values of the study population

\begin{tabular}{llll}
\hline & SLE patients & Controls & p-value \\
\hline Subjects, $\mathrm{n}$ & 54 & 20 & \\
Females, n $(\%)$ & $49(90.7)$ & $18(90)$ & - \\
Age (y) & & & \\
$\quad$ average \pm SD & $43.7 \pm 12.4$ & $45.2 \pm 10.6$ & $n s$ \\
CV risk factors, n (\%) & $30(55.5)$ & $5(25)$ & $n s$ \\
$\quad$ Smokers & $9(16.6)$ & $5(25)$ & $n s$ \\
Valvulopathies, n (\%) & $10(18.5)$ & $3(16.6)$ & $n s$ \\
CVD, n (\%) & $3(5.4)$ & 0 & $n s$ \\
sPAP (mmHg) & & & 0.0003 \\
$\quad$ average \pm SD & $28.2 \pm 11.9$ & $16.2 \pm 6.6$ & \\
Age at diagnosis (years) & & & - \\
$\quad$ median, range & $34.0(17-58)$ & - & - \\
$\begin{array}{l}\text { Disease duration (y) } \\
\quad \text { median (range) }\end{array}$ & $7.0(0.3-43)$ & - & \\
\hline frmer or current smokers & & &
\end{tabular}

$¥$ former or current smokers

$\mathrm{CV}=$ cardiovascular; $\mathrm{CVD}=$ cardiovascular disease (ischemic heart disease, stroke/ transient ischemic attack, peripheral arterial disease); $\mathrm{sPAP}=$ systolic pulmonary arterial pressure

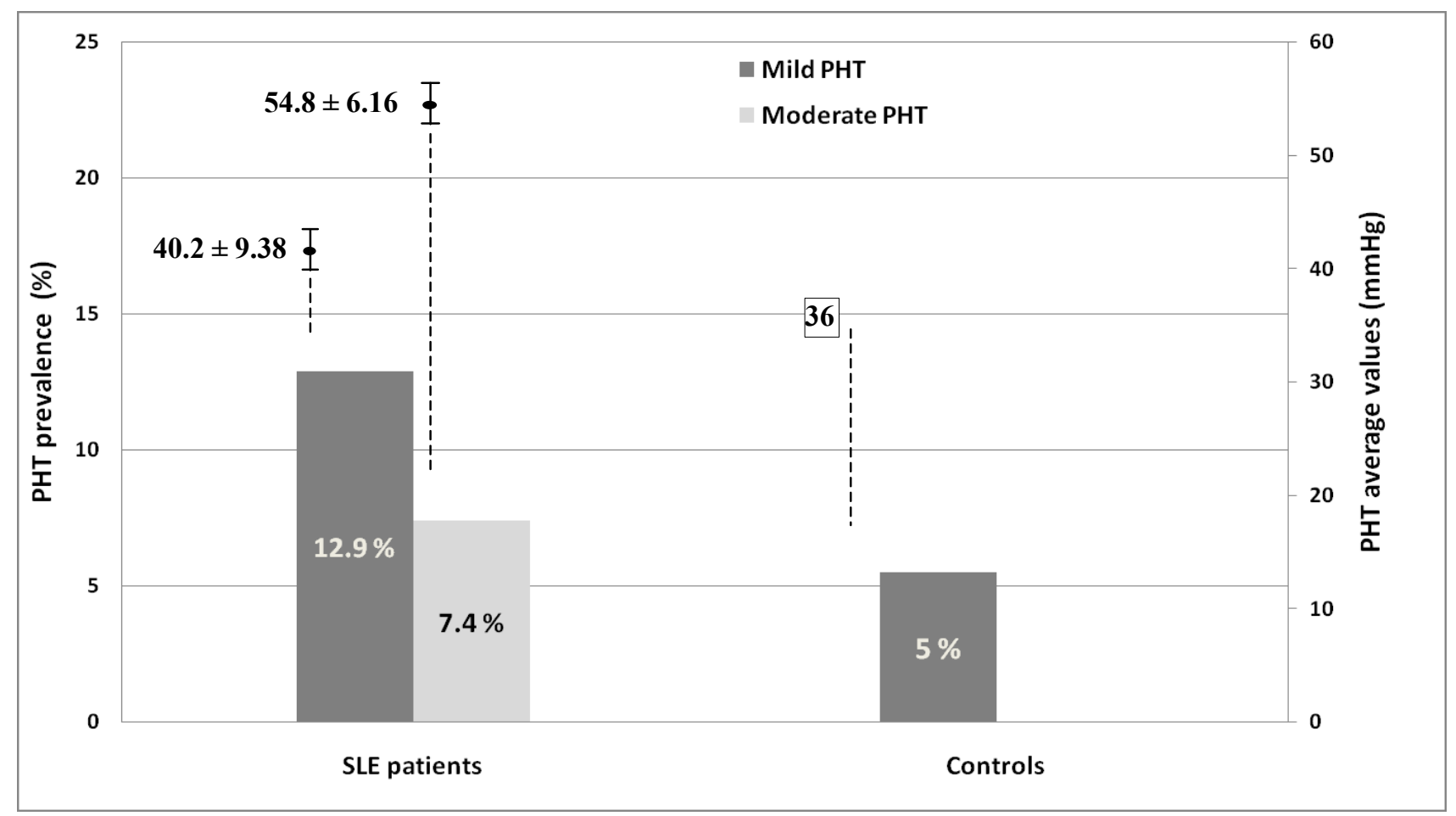

Figure 1. Prevalence and severity of pulmonary hypertension in the study population. Values represent average sPAP $( \pm \mathrm{SD})$. sPAP=systolic pulmonary arterial pressure; $\mathrm{PHT}=$ pulmonary hypertension; $\mathrm{SLE}=$ systemic lupus erythematosus 
Table II. Comparison between the clinical and laboratory features of SLE patients with PAH versus those without PAH.

\begin{tabular}{|c|c|c|c|}
\hline & $\begin{array}{l}\text { SLE patients } \\
\text { with PAH }\end{array}$ & $\begin{array}{l}\text { SLE patients } \\
\text { without PAH }\end{array}$ & p-value \\
\hline Age $(y)$, mean \pm SD & $49.6 \pm 12.32$ & $42.2 \pm 12.12$ & ns \\
\hline $\begin{array}{r}\text { Disease duration (y), median } \\
\text { (range) }\end{array}$ & $\begin{array}{l}10 \\
(1.3-43)\end{array}$ & $\begin{array}{l}6 \\
(0.3-23)\end{array}$ & ns \\
\hline $\mathrm{CV}$ risk factors, $\mathrm{n}(\%)$ & $8(72.7)$ & $22(51)$ & ns \\
\hline Smokers ${ }^{*}$ & $2(18.1)$ & $7(16.2)$ & $n s$ \\
\hline Dyspnea, n (\%) & $5(45)$ & $11(25)$ & $n s$ \\
\hline Other symptoms ${ }^{\#}$ & $3(27.2)$ & $8(18)$ & $n s$ \\
\hline Raynaud's phenomenon, n (\%) & $4(36.3)$ & $13(30.2)$ & ns \\
\hline Associated AI conditions ${ }^{\S}, \mathrm{n}(\%)$ & $1(9.1)$ & $4(9.3)$ & $n s$ \\
\hline Sjögren's syndrome, n (\%) & $2(18.1)$ & $3(6.9)$ & $n s$ \\
\hline Vasculitis, n (\%) & $2(18.1)$ & $2(4.65)$ & $n s$ \\
\hline $\begin{array}{r}\text { Musculoskeletal involvement, } \\
\mathrm{n}(\%)\end{array}$ & $9(81.8)$ & $39(90.6)$ & $n s$ \\
\hline Lupus nephritis, n (\%) & $2(18.1)$ & $8(18.6)$ & ns \\
\hline $\begin{array}{r}\text { Neuropsychiatric involvement, } \\
\mathrm{n}(\%)\end{array}$ & $3(27.2)$ & $6(13.9)$ & $n s$ \\
\hline Thromboembolic events, n (\%) & $8(72.7)$ & $1(2.3)$ & 0.0067 \\
\hline Anti- ${ }_{\mathrm{ds}} \mathrm{DNA}, \mathrm{n}(\%)$ & $3(27.2)$ & $8(18.6)$ & ns \\
\hline $\mathrm{aPL}\left(\mathrm{aCL} / \beta_{2}-\mathrm{GPI} / \mathrm{LAC}\right)$ & $7(63.6)$ & 7 (15.9) & 0.039 \\
\hline SLICC $\geq 1, \mathrm{n}(\%)$ & $8(72.7)$ & $6(13.9)$ & 0.001 \\
\hline
\end{tabular}

Former or current smokers;

\# potentially related with PAH (chest pain/discomfort, palpitations, fatigue, syncope)

$\S$ except for vasculitis or Sjogren's syndrome: thyroiditis, autoimmune hepatitis, ulcerohemorrhagic rectocolitis

$\mathrm{PAH}=$ pulmonary arterial hypertension; $\mathrm{CV}=$ cardiovascular; $\mathrm{AI}=$ autoimmune; $\mathrm{SLICC}=$ the Systemic Lupus International Collaborating Clinics damage index; ${ }_{\mathrm{ds}} \mathrm{DNA}=\mathrm{double-}$ stranded DNA; aPL=antiphospholipid antibodies; $\mathrm{aCL}=$ anticardiolipin; $\beta_{2}-\mathrm{GPI}=\beta_{2^{-}}$ glycoprotein I; LAC=lupus anticoagulant

Table III. Echocardiographic parameters in the two subgroups of patients, with and without PAH

\begin{tabular}{|c|c|c|c|}
\hline & $\begin{array}{l}\text { SLE patients } \\
\text { with PAH }\end{array}$ & $\begin{array}{l}\text { SLE patients } \\
\text { without PAH }\end{array}$ & p-value \\
\hline $\begin{array}{l}\mathrm{sPAP}(\mathrm{mmHg}), \\
\text { mean } \pm \mathrm{SD}\end{array}$ & $45.54 \pm 8.2$ & $22.0 \pm 5.06$ & 0.021 \\
\hline LVEF $(\%)$ & $63.9 \pm 10.35$ & $58.9 \pm 10.3$ & ns \\
\hline Valve regurgitation ${ }^{€}, \mathrm{n}(\%)$ & $4(36.3)$ & $6(13.95)$ & ns \\
\hline Pericardial effusion, $\mathrm{n}(\%)$ & $4(36.3)$ & $9(20.9)$ & ns \\
\hline $\begin{array}{l}\text { Diastolic dysfunction, } \\
\mathrm{n}(\%)\end{array}$ & $10(90.9)$ & $11(25.5)$ & 0.008 \\
\hline
\end{tabular}




\section{Discussion}

This study is one of the few carried out in Romania that monitored the features of PAH in a population of patients with SLE and showed that its prevalence $(20.3 \%)$ is significantly higher than in the general population and falls within the middle range of values reported by other similar studies $(4.1 \%$ - 43\%) $(9,10,11)$. Prabu, in an analysis conducted on 288 patients with a prevalence at the lower limit of the reported value ranges, of $4.2 \%$ (10), explains these differences by the different size of the study population, by the modality of pressure gradient assessment across the tricuspid valve (the regurgitant jet cannot be evidenced in $15-60 \%$ of patients) and RA pressure, as well as by the different patient registries, the proportion of patients with advanced disease stages being higher in tertiary centers. In our study, tricuspid regurgitation could not be detected in $22.2 \%$ of the patients, and this could lead to under estimation of PAH prevalence (4); in the present study however the higher $\mathrm{PAH}$ prevalence as detected by ultrasound could result from sPAP overestimation, which is possible especially in dehydrated patients or if one doesn't take into account respiratory variations of tricuspid blood flow as well as if excessive Doppler gain settings. We had in mind this factors and tried to counteract them, but definitively the right heart catheterization could establish the accurate diagnosis, if potential benefits outweigh costs and risks of the procedure.

Systolic PAP values in the subgroup of patients with PAH were comparable to those obtained by REVEAL (45.54 vs $46.6 \mathrm{mmHg}$ ) or other studies $(3,9)$; REVEAL also shown that PAH in SLE does not have a significantly more favorable hemodynamic profile (pulmonary vascular resistances, right heart pressures) than $\mathrm{SSc}$, but the evolution of the latter is more severe, so that according to some opinions, in the absence of treatment it leads to death in $50 \%$ of the cases within two years from the onset of symptoms (12). This suggests the presence of several pathogenic pathways with different dominance in the two diseases, and the first indications might be provided by necropsy studies describing thromboembolic lesions $(7.8 \%$ of patients with SLE) on the one hand, and plexiform lesions (5-6\% of patients) on the other hand (13), which are focal proliferations of endothelial cells, surrounded by myofibroblasts, smooth muscle cells and matrix connective tissue (14). These lesions form the substrate of the socalled plexogenic pulmonary arterial hypertension (15), found in IPAH, PAH associated with SSc, as well as in some aforementioned cases of PAH associated with SLE. Lesions have also been described in PAH accompanying HIV infection with low CD4 lymphocyte activity; this correlation provides an additional clue for the "immunogenic" theory of the arterial wall's "plexiform" damage that could be associated with a more severe evolution of PAH in SSc and in some SLE patients (11).

Another group of lupus patients, with a milder evolution of PAH might have in situ thrombotic or thromboembolic lesions as a dominant injury of the vascular bed, assumption supported by the positive association found in our study and other studies $(3,10,15)$, between $\mathrm{PAH}$ and thrombotic events or between $\mathrm{PAH}$ and the antiphospholipid antibodies (aPL). Anticoagulants and "classical" vasodilators (calcium channel blockers, nitrates) together with immunosuppressive therapy probably provides a better evolution of these patients (14).

Finally, the vasoconstriction resulting from both impaired production of vasodilators and overexpression of vasoconstriction mediators, is described in most PAH cases, either as an additional mechanism or as an early alteration of the pulmonary aterial bed, as seen in PAH secondary to respiratory diseases. It should be expected that in SLE (and other CTDs), PAH will accompany the vasospastic phenomena in other territories, such as Raynaud's phenomenon; however, this association is questionable, being present in some studies $(9,16)$, absent or without statistical significance in studies including larger patient populations $(3,10)$. In our study, Raynaud's phenomenon was present in about 
one third of the patients (compared to $45 \%$ in REVEAL), but its frequency was not different in the subgroup of patients with PAH. Certainly, the size of the group was small, but as we mentioned before, larger studies also failed to demonstrate this correlation, probably because of the lower role of this vasospastic component in the pathogenesis of $\mathrm{PAH}$, or because of different vasospasm induction pathways (17) in the two systemic and pulmonary (micro)circulation types.

Another correlation that was not evidenced is that between the presence of PAH and the disease duration, as PAH progresses in SLE, even if less rapid than in SSc (11). There is a difference of the median values (10 vs 6 years), but it didn't reach the statistical significance. This could be mainly explained by the small size of the group, and probably, by the relatively short time elapsed from diagnosis (the median duration of the disease was 7 years in the whole group), and certainly, by the efficacy of the treatment prescribed in a tertiary rheumatology center.

An unsolved issue is pointing toward the relationship diastolic dysfunction pulmonary hypertension. This association has long been described in patient with diastolic heart failure, although its prevalence is still poorly defined. The studies conducted in this direction have shown that PAH related to diastolic dysfunction tends to occur in the elderly patients, female gender, with larger size of left atrium (LA), a higher prevalence of atrial fibrillation (AF) and mitral regurgitation (18). In our study, of these features, none was significantly noted in the PAH group (data on AF and LA dimensions not shown). That's why we assume that in our patients both pulmonary hypertension and diastolic dysfunction are the consequence of the SLE pathogenic mechanisms and not two consecutive steps directly related each to other (as they are in typical heart failure patients). Moreover, the PAH group had significantly higher values of the damage index (even if there was no correlation with renal or neurological involvement, probably again due to the small size of the group), which shows that the same factors that lead to the impairment of other systems up to the clinical stage, also result in or facilitate the occurrence of PAH in this patients.

\section{Limits of the study}

The main limit is the small size of the study population, which might explain why some results didn't reach statistical significance. Secondly the absence of pulmonary pressures conformation by right heart catheterization may have allowed a possible overestimation of $\mathrm{PAH}$ prevalence. However the underestimation of SPAP by ultrasound is much common than overestimation, and we tried to take in account and to avoid the sources of error for the latter. Finally the treatment either at the time of evaluation, or the long-term therapeutic regimens, were not taken into consideration. The resulting groups would have been too small to be statistically analyzed. The fact that all patients came from the same hospital, with standard treatment protocols, makes us assume that the results were not significantly influenced.

\section{Conclusions}

PAH diagnosed by Doppler ultrasound is found in a significant proportion of patients with SLE; in the majority of the cases, it is asymptomatic and falls within severity grade I. The presence of PAH is correlated with the presence of APS syndrome, the damage index, and diastolic dysfunction; these observations might allow the selection of subgroups of patients to be referred for ultrasound for the early detection of PAH. Further studies are required to evidence the progression rate of PAH and possibly, the efficacy of pulmonary vasodilators for improving the prognosis of these 


\section{References}

1. Nazzareno G, Hoeper MM, Humbert M et al. Guidelines for the diagnosis and treatment of pulmonary hypertension. Eur Heart J 2009; 30: 2493-2537.

2. Chung SM, Lee CK, Lee E, Yoo B, Lee SD, Moon H-B. Clinical aspects of pulmonary hypertension in patients with systemic lupus erythematosus and in patients with idiopathic pulmonary arterial hypertension. Clinical Rheumatology 2006;25(6):866-72.

3. Chung L, Liu J, Parsons L et al. Characterization of Connective Tissue Disease-Associated Pulmonary Arterial Hypertension From REVEAL.Chest 2010; 138(6):1383-1394.

4. Janda S, Shahidi N, Gin K, Swiston J. Diagnostic accuracy of echocardiography for pulmonary hypertension: a systematic review and meta-analysis. Heart 2011;97:612-622.

5. Litwin SE. Noninvasive Assessment of Pulmonary Artery Pressures - Moving Beyond Tricuspid Regurgitation Velocities. Circ Cardiovasc Imaging 2010;3:132-133.

6. Tan EM, Cohen AS, Fries JF, et al. The 1982 revised criteria for the classification of systemic lupus erythematosus. Arthritis Rheum 1982;25:1271-7.

7. Selwyn P, Wong MD, Catherine M, Otto MD. Echocardiographic Findings in Acute and Chronic Pulmonary Disease. In: Textbook of Clinical Echocardiography. Ed. Otto CM, Catherine M, 5th Edition. Elsevier Saunders 2013: 741-760.

8. Nagueh et al. Recommendations for the Evaluation of Left Ventricular Diastolic Function by Echocardiography. European Journal of Echocardiography 2009;10: 165-193.

9. Kamel SR, Omar GM, Darwish AF, Asklany HT, Ellabban AS. Asymptomatic Pulmonary Hypertension in Systemic Lupus Erythematosus. Clinical Medicine
Insights: Arthritis and Musculoskeletal Disorders 2011; 4:77-86.

10. Prabu A, Patel K, Yee CS, Nightingale P, Situnayake RD, Thickett DR, et al. Prevalence and risk factors for pulmonary arterial hypertension in patients with lupus. Rheumatology 2009;48(12):150611.

11. Teng J, Zhang W. Systemic lupus erythematosus associated pulmonary arterial hypertension: clinical analysis of 91 cases. Zhonghua Yi Xue Za Zhi 2014;94(13):969-72.

12. Tamborrini G, Distler O. Update in pulmonary hypertension associated with connective tissue diseases - a systematic literature review. Dtsch Med Wochenschr 2008;133(6): S199-202.

13. Quadrelli SA, Alvarez C, Arce SC, Paz L, Sarano J, Sobrino EM, et al. Pulmonary involvement of systemic lupus erythematosus: analysis of 90 necropsies. Lupus 2009; 18(12):1053-60.

14. Rich S, McLaughlin VV. Pulmonary hypertension. In: Braunvald's Heart Disease: A Textbook of Cardiovascular Medicine, 9th edition. Saunders Elsevier 2011:1883-914

15. Asherson RA, Cervera R. Pulmonary hypertension, antiphospholipid antibodies, and syndromes. Clin Rev Allergy Immunol 2007;32(2):153-8.

16. Kasparian A, Floros A, Gialafos E, Kanakis M, Tassiopoulos S, Kafasi N, et al. Raynaud's phenomenon is correlated with elevated systolic pulmonary arterial pressure in patients with systemic lupus erythematosus. Lupus 2007;16(7):505-8.

17. Galiè N, Manes A, Farahani KV et al . Pulmonary arterial hypertension associated to connective tissue diseases. Lupus 2005;14(9):713 - 717 .

18. Fitzgibbons TP, Bouchard JL, Meyer TE, Aurigemma GP. Prevalence of Pulmonary Hypertension in Elderly Patients with Diastolic Heart Failure. Journal of Cardiac Failure 2010; 13(6):S93. 